\title{
Two dimensional dynamical systems which admit Lie and Noether symmetries
}

\author{
Michael Tsamparlis* and Andronikos Paliathanasis ${ }^{\dagger}$ \\ Faculty of Physics, Department of Astrophysics - Astronomy - Mechanics, \\ University of Athens, Panepistemiopolis, Athens 157 83, GREECE
}

\begin{abstract}
We consider a dynamical system moving in a Riemannian space and prove two theorems which relate the Lie point symmetries and the Noether symmetries of the equation of motion, with the special projective group and the homothetic group of the space respectively. These theorems are used to classify the two dimensional Newtonian dynamical systems, which admit Lie point/Noether symmetries. The results of the study i.e. expressions of forces / potentials, Lie symmetries, Noether vectors and Noether integrals are presented in the form of tables for easy reference and convenience. Two cases are considered, Hamiltonian and non-Hamiltonian systems. The results are used to determine the Lie / Noether symmetries of two different systems. The Kepler - Ermakov system, which in general is non-conservative, and the conservative system with potential similar to the Hènon Heiles potential. As an additional application, we consider the scalar field cosmologies inFRW background with no matter, and look for the scalar field potentials for which the resulting cosmological models are integrable. It is found that the only integrable scalar field cosmologies are defined by the exponential and the Unified Dark Matter potential. It is to be noted that in all aforementioned applications the Lie / Noether symmetry vectors are found by simply reading the appropriate entry in the relevant tables.
\end{abstract}

Keywords: Dynamical systems, Lie point symmetries, Special projective group, Noether symmetries, Homothetic motions, scalar field cosmology, Kepler, Ermakov, Hènon Heiles potential

PACS - numbers: 2.40.Hw, 4.20.-q, 4.20.Jb, 04.20.Me, 03.20.+i, 02.40.Ky

\section{Introduction}

The study of Lie point symmetries of a given system of ODEs consists of two steps (a) the determination of the conditions, which the components of the Lie symmetry vectors must satisfy and (b) the solution of the system of these conditions. Step (a) is formal and it is outlined in e.g. 1, 2]. These conditions can be quite involved, but today it is possible to use algebraic computing programs to derive them. Therefore the essential part of

\footnotetext{
*Email: mtsampa@phys.uoa.gr

$\dagger$ Email: anpaliat@phys.uoa.gr
} 
the work is the second step. For a small number of equations (say up to three) one can possibly employ again computer algebra to look for a solution of the system. However for a large number of equations such an attempt is prohibitive and one has to go back to traditional methods to determine the solution.

The purpose of the present work is to provide an alternative way to solve the system of Lie point symmetry conditions for the second order equations of the form:

$$
\ddot{x}^{i}+\Gamma_{j k}^{i} \dot{x}^{j} \dot{x}^{k}=F^{i} .
$$

Here $\Gamma_{j k}^{i}\left(x^{r}\right)$ are general functions, a dot over a symbol indicates derivation with respect to the parameter $s$ along the solution curves and $F^{i}\left(x^{j}\right)$ is a $C^{\infty}$ vector field. This type of equations is important, because it contains the equations of motion of a dynamical system in a Riemannian space, in which the functions $\Gamma_{j k}^{i}\left(x^{r}\right)$ are the connection coefficients of the metric, $s$ being an affine parameter along the trajectory. In the following we assume this identification of $\Gamma_{j k}^{i}, 1$.

The key idea, which is proposed here, is to express the system of Lie symmetry conditions of (1) in a Riemannian space in terms of collineation (i.e. symmetry) conditions of the metric. If this is achieved, then the Lie point symmetries of (1) will be related to the collineations of the metric, hence their determination will be transferred to the geometric problem of determining the generators of a specific type of collineations of the metric. One then can use of existing results of Differential Geometry on collineations to produce the solution of the Lie symmetry problem.

The natural question to ask is: If the Lie symmetries of the dynamical systems moving in a given Riemannian space are from the same set of collineations of the space, how will one select the Lie symmetries of a specific dynamical system? The answer is as follows. The left hand side of Equation (11) contains the metric and its derivatives and it is common to all dynamical systems moving in the same Riemannian space. Therefore geometry (i.e. collineations) enters in the left hand side of (1) only. A dynamical system is defined by the force field $F^{i}$, which enters into the right hand side of (10) only. Therefore, there must exist constraint conditions, which will involve the components of the collineation vectors and the force field $F^{i}$, which will select the appropriate Lie symmetries for a specific dynamical system. Indeed Theorem 1 (see section 3) relates the Lie point symmetry generators of (11) with the elements of the special projective Lie algebra of the space where motion occurs, and provides these necessary constraint conditions. A similar approach can be found in [3, 4 , and for geodesic equations in [5, 6].

What has been said for the Lie point symmetries of (1) applies also to Noether symmetries. The Noether symmetries are Lie point symmetries which satisfy the constraint

$$
X^{[1]} L+L \frac{d \xi}{d t}=\frac{d f}{d t} .
$$

Theorem 2 (see section 4) relates the generators of Noether symmetries of (1) with the homothetic algebra of the metric and provides the required constraint conditions.

Before we continue, we recall briefly some definitions from Riemannian geometry. A collineation in a Riemannian space is a vector field $X^{i}$ which satisfies an equation of the form

$$
\mathcal{L}_{X} \mathbf{A}=\mathbf{B}
$$

\footnotetext{
${ }^{1}$ Of course it is possible to look for a metric for which a given set of $\Gamma_{j k}^{i}$ are the connection coefficients, or, even avoid the metric altogether. However we shall not attempt this in the present work. For such an attempt see [7.
} 
where $\mathcal{L}_{X}$ denotes Lie derivative, $\mathbf{A}$ is a geometric object (not necessarily a tensor) defined in terms of the metric and its derivatives (e.g. connection coefficients, Ricci tensor, curvature tensor etc.) and $\mathbf{B}$ is an arbitrary tensor with the same tensor indices as A. In Table 1 we show examples of collineations which we shall use in the present work

Table 1: Collineations of space

\begin{tabular}{|l|c|l|}
\hline Collineation & A & B \\
\hline Killing vector (KV) & $g_{i j}$ & 0 \\
\hline Homothetic vector (HV) & $g_{i j}$ & $\psi g_{i j}, \psi_{, i}=0$ \\
\hline Conformal Killing vector (CKV) & $g_{i j}$ & $\psi g_{i j}, \psi_{, i} \neq 0$ \\
\hline Affine Collineation (AC) & $\Gamma_{j k}^{i}$ & 0 \\
\hline Projective collineation (PC) & $\Gamma_{j k}^{i}$ & $2 \phi_{(, j} \delta_{k)}^{i}, \phi,,_{i} \neq 0$ \\
\hline Special Projective collineation $(\mathrm{SPC})$ & $\Gamma_{j k}^{i}$ & $2 \phi_{(, j} \delta_{k)}^{i}, \phi,,_{i} \neq 0$ and $\phi,{ }_{j k}=0$ \\
\hline
\end{tabular}

From Differential Geometry we know that the special projective Lie algebra of the Euclidian space $E^{n}$ consists of the vector fields of Table 2

Table 2: Collineations of Euclidean space $E^{n}$

\begin{tabular}{|l|l|l|}
\hline Collineation & Gradient & Non-gradient \\
\hline Killing vectors $(\mathrm{KV})$ & $\mathbf{S}_{I}=\delta_{I}^{i} \partial_{i}$ & $\mathbf{X}_{I J}=\delta_{[I}^{j} \delta_{j]}^{i} x_{j} \partial_{i}$ \\
\hline Homothetic vector (HV) & $\mathbf{H}=x^{i} \partial_{i}$ & \\
\hline Affine Collineation (AC) & $\mathbf{A}_{I J}=x_{J} \delta_{I}^{i} \partial_{i}$ & \\
\hline Special Projective collineation $(\mathrm{SPC})$ & & $\mathbf{P}_{I}=S_{I} \mathbf{H}$. \\
\hline
\end{tabular}

where the indices $I, J=1,2, \ldots, n$.

In the following sections we apply Theorem 1 and Theorem 2 to determine all Newtonian dynamical systems with two degrees of freedom, moving under the action of a general force $F^{i}$, which admit Lie and Noether symmetries. We also derive for each case the relevant Noether function and the corresponding Noether integral. The case $F^{i}=0$ corresponds to the Lie point symmetries of the geodesic equations and has been considered in 6]. The case of a conservative force has been addressed previously by Sen 8 and more recently by Damianou et al [9. As it will be shown both treatments are incomplete. We demonstrate the use of the results in two cases. The non-conservative Kepler - Ermakov system [10, 11, 12, and the case of the Hènon Heiles type potentials [13, 14. In both cases we recover and complete the existing results. Finally we consider the interesting case of scalar field cosmologies, which is reduced to a system of two equations of motion in a flat two dimensional Lorentzian space, and show that the only scalar field potentials which lead to an integrable cosmological model, are the exponential potential [19] and the Unified Dark Matter (UDM) potential [17, 18. 


\section{The Lie point symmetry conditions}

Using the standard approach we derive the Lie point symmetry conditions for equation (11). We prefer to use the more geometric method outlined in 2 rather that the more algebraic (but equivalent) derivation given e.g. in [1].

We write the system of ODEs (11) in the form $\ddot{x}^{i}=\omega^{i}(x, \dot{x}, t)$ where

$$
\omega^{i}(x, \dot{x}, t)=-\Gamma_{j k}^{i}(x) \dot{x}^{j} \dot{x}^{k}-F^{i} .
$$

The associated linear operator defined by this system of ODEs is

$$
\mathbf{A}=\frac{\partial}{\partial t}+\dot{x}^{i} \frac{\partial}{\partial x^{i}}+\omega^{i}\left(t, x^{j}, \dot{x}^{j}\right) \frac{\partial}{\partial \dot{x}^{i}} .
$$

The condition for a Lie symmetry for the system of equations is 2 ]

$$
\left[\mathbf{X}^{[1]}, \mathbf{A}\right]=\lambda\left(x^{j}\right) \mathbf{A}
$$

where $X^{[1]}$ is the first prolongation of the symmetry vector $X=\xi(t, x) \partial_{t}+\eta^{i}(t, x) \partial_{x^{i}}$ defined as follows

$$
\mathbf{X}^{[1]}=\xi(t, x, \dot{x}) \partial_{t}+\eta^{i}(t, x, \dot{x}) \partial_{x^{i}}+G^{[1] i} \partial_{\dot{x}^{i}} .
$$

$G^{[1] i}$ is the component of the first prolongation $\mathbf{X}^{[1]}$ along $\partial_{\dot{x}^{i}}$. It is a standard result [2] that (6) leads to the three conditions:

$$
\begin{aligned}
-\mathbf{A} \xi & =\lambda \\
G^{[1] i} & =\mathbf{A} \eta^{i}-\dot{x}^{i} \mathbf{A} \xi \\
X^{[1]}\left(\omega^{i}\right)-\mathbf{A}\left(G^{[1] i}\right) & =-\omega^{i} \mathbf{A} \xi .
\end{aligned}
$$

For any function $f\left(t, x^{i}\right), \mathbf{A} f=d f / d t$, where $d f / d t=f_{, t}+f_{, i} \dot{x}^{i}$ is the total derivative of $f$. Using this result we write the symmetry conditions as

$$
\begin{aligned}
\lambda & =-\frac{d \xi}{d t} \\
G^{[1] i} & =\frac{d \eta^{i}}{d t}-\dot{x}^{i} \frac{d \xi}{d t} \\
X^{[1]}\left(\omega^{i}\right)-A\left(G^{[1] i}\right) & =-\omega^{i} \frac{d \xi}{d t} .
\end{aligned}
$$

We note that condition (12) defines the first prolongation $G^{[1] i}$. Condition (11) gives the factor $\lambda$. Therefore the essential condition for a Lie point symmetry is equation (13).

We introduce the second prolongation $G^{[2] i}$ of $\mathbf{X}$ with the formula

$$
G^{[2] i}\left(t, x^{i}, \dot{x}^{i}\right)=\frac{d G^{[1] i}}{d t}-\ddot{x}^{i} \frac{d \xi}{d t}=A\left(G^{[1] i}\right)-\omega^{i} \frac{d \xi}{d t} .
$$

Then the condition for a Lie symmetry becomes [2]:

$$
G^{[2] i}=X^{[1]} \omega^{i} .
$$


After a rather long but standard calculation we find that the Lie point symmetry conditions of (11) are

$$
\begin{gathered}
L_{\eta} F^{i}+2 \xi{ }_{, t} F^{i}+\eta^{i},{ }_{t t}=0 \\
\left(\xi_{, k} \delta_{j}^{i}+2 \xi,_{j} \delta_{k}^{i}\right) F^{k}+2 \eta^{i}{ }_{t \mid j}-\xi_{, t t} \delta_{j}^{i}=0 \\
L_{\eta} \Gamma_{(j k)}^{i}=2 \xi_{, t(j} \delta_{k)}^{i} \\
\xi_{(, i \mid j} \delta_{r)}^{k}=0 .
\end{gathered}
$$

We note that for $F^{i}=0$ we recover the Lie point symmetry conditions of the geodesic equations (see [6])

$$
\begin{aligned}
\eta^{i}, t t & =0 \\
2 \eta^{i}{ }_{t \mid j}-\xi_{, t t} \delta_{k}^{i} & =0 \\
L_{\eta} \Gamma_{j k}^{i}-2 \xi_{, t(j} \delta_{k)}^{i} & =0 \\
\xi_{(, j \mid k} \delta_{d)}^{i} & =0 .
\end{aligned}
$$

\section{Lie point symmetries and collineations}

Following a similar approach as in [6] we express the Lie point symmetry conditions (16) - (19) in terms of the collineations of the metric.

Equation (19) means that $\xi_{, j}$ is a gradient Killing vector (KV) of $g_{i j}$. This implies that the metric $g_{i j}$ is decomposable. Equation (18) means that $\eta^{i}$ is a projective collineation of the metric with projective function $\xi_{, t}$. The remaining two equations are the constraint conditions, which relate the components $\xi, n^{i}$ of the Lie point symmetry vector with the vector $F^{i}$. Equation (16) gives?

$$
\left(L_{\eta} g^{i j}\right) F_{j}+g^{i j} L_{\eta} F_{j}+2 \xi_{, t} g^{i j} F_{j}+\eta_{, t t}^{i}=0
$$

This equation restricts $\eta^{i}$ further because it relates it directly to the metric symmetries. Finally equation (17) gives

$$
-\delta_{j}^{i} \xi_{, t t}+\left(\xi_{, j} \delta_{k}^{i}+2 \delta_{j}^{i} \xi_{, k}\right) F^{k}+2 \eta_{, t j}^{i}+2 \Gamma_{j k}^{i} \eta_{, t}^{k}=0 .
$$

We conclude that the Lie symmetry equations are equations (24), (25) where $\xi(t, x)$ is a gradient $\mathrm{KV}$ of the metric $g_{i j}$ and $\eta^{i}(t, x)$ is a special Projective of the metric $g_{i j}$ with projective function $\xi_{, t}$. We state the solution of the system of equations (16) - (19) as Theorem 1,

Theorem 1 The Lie point Symmetries of the equations of motion of an autonomous system with force function $F^{j}\left(x^{i}\right)$, in a general Riemannian space with metric $g_{i j}$, namely

$$
\ddot{x}^{i}+\Gamma_{j k}^{i} \dot{x}^{j} \dot{x}^{k}=F^{i}
$$

are given in terms of the generators $Y^{i}$ of the special projective Lie algebra of the metric $g_{i j}$ as follows.

One Lie symmetry vector is $\partial_{t}$ (autonomous equation of motion).

Case A: The force is not necessarily conservative

\footnotetext{
${ }^{2} L_{\eta} V_{b}=V_{, b c} \eta^{c}+\eta^{c},{ }_{b} V_{, c}$
} 
Case A1.

$Y^{i}$ is an element of the Affine algebra of the metric.

The Lie symmetries are:

$$
\mathbf{X}=\left(\frac{1}{2} d_{1} a_{1} t+d_{2}\right) \partial_{t}+a_{1} Y^{i} \partial_{i}
$$

where $a_{1}$ and $d_{1}$ are constants, provided the force satisfies the condition:

$$
L_{Y} F^{i}+d_{1} F^{i}=0 .
$$

Case A2.

$Y^{i}$ is a gradient $K V$ or $H V$ (if it exists) and $Y^{i} \neq F^{i}$.

The Lie symmetries are:

$$
\mathbf{X}=2 \psi \int T(t) d t \partial_{t}+T(t) Y^{i} \partial_{i},
$$

where the function $T(t)$ is the solution of the equation

$$
T_{, t t}=a_{1} T
$$

provided the force $F^{i}$ satisfies the condition

$$
L_{\mathbf{Y}} F^{i}+4 \psi F^{i}+a_{1} Y^{i}=0 .
$$

Case $A 3$

$Y^{i}$ is a special $P C$.

In this case the Lie symmetry vectors are (the index $J$ counts the gradient KVs)

$$
\mathbf{X}_{J}=\left(C(t) S_{J}+D(t)\right) \partial_{t}+T(t) Y^{i} \partial_{i},
$$

where the functions $C(t), T(t), D(t)$ are solutions of the system of simultaneous equations

$$
\frac{D_{, t}}{T}=\frac{1}{2} d_{1} \quad, \quad \frac{T_{, t t}}{T}=a_{1}, \frac{T_{, t}}{C}=c_{2}, \frac{D_{, t t}}{C}=d_{c}, \frac{C_{, t}}{T}=a_{0},
$$

provided the force satisfies the conditions

$$
\begin{aligned}
L_{Y} F^{i}+2 a_{0} S F^{i}+d_{1} F^{i}-a_{1} Y^{i} & =0 \\
\left(S_{, k} \delta_{j}^{i}+2 S,_{j} \delta_{k}^{i}\right) F^{k}-\left(2 Y^{i} ; j-a_{0} S \delta_{j}^{i}\right) c_{2}+d_{c} \delta_{j}^{i} & =0 .
\end{aligned}
$$

Case B: The force is conservative and $F^{i}=-V^{, i}$.

In addition to the cases of Case A we have the following extra cases.

Case B1.

$Y^{i}$ is a gradient $H V$ and $Y^{i}=\kappa V^{, i}$, where $\kappa$ is a constant.

In this case the potential is the function generating the gradient $H V$ of the metric and the Lie symmetry vectors are

$$
\mathbf{X}=D(t) \partial_{t}+T(t) V^{, i} \partial_{i}
$$


where the functions $D(t), T(t)$ are the solutions of the system of simultaneous equations

$$
\begin{aligned}
& D_{, t t}-2 \psi T_{, t}=0 \\
& \kappa T_{, t t}+2 D_{, t}=0 .
\end{aligned}
$$

Case B2.

$Y^{i}$ is a special $P C$ and $Y_{J}^{i}=\lambda S_{J} V^{, i}$, where $V^{, i}$ is a gradient $H V$ and $S_{J}^{, i}$ is a gradient $K V$.

The Lie symmetry vectors are:

$$
X_{J}=\left(C(t) S_{J}+d_{1}\right) \partial_{t}+T(t) \lambda S_{J} V^{, i} \partial_{i}
$$

where the functions $C(t)$ and $T(t)$ are computed from:

$$
\frac{T, t t}{T}+2 \frac{C_{, t}}{T}=\lambda_{1} \quad, \frac{T_{, t}}{C}=\lambda_{2}, C_{, t}=a_{0} T
$$

provided the potential function satisfies the conditions

$$
\begin{aligned}
L_{\mathbf{Y}_{J}} V^{, i}+\lambda_{1} S_{J} V^{, i} & =0 \\
{\left[\lambda_{2}\left(2 \lambda-a_{0}\right)+\lambda_{1}\right] S_{J} \delta_{j}^{i}+2\left(1+\lambda \lambda_{2}\right) S_{J, j} V^{, i} } & =0 .
\end{aligned}
$$

Conditions (40) and (41) is possible to be written in a simpler form. Let $H=k V^{, i}(k \neq 0)$ then $Y^{i}=$ $\lambda S_{J} V^{, i}=\frac{\lambda}{k} S_{J} H$.

Then condition (40) gives (we have changed $\lambda_{1}$ to $\lambda_{J}$ ):

$$
H\left(\ln S_{J}\right)=\frac{\lambda_{J}}{\lambda} k
$$

which is a condition on the gradient $\mathrm{HV} H^{i}$.

Concerning the second condition (41) we find:

$$
2\left(1+\lambda \lambda_{2}\right)\left(\ln S_{J}\right),{ }_{j} H^{i}=-k\left[\lambda_{2}\left(2 \lambda-a_{0}\right)+\lambda_{1}\right] \delta_{j}^{i} .
$$

This condition is also a constraint between the gradient functions $S_{J}, H$ for all $J$.

\section{The Noether symmetries of an autonomous conservative dynam- ical system moving in a Riemannian space}

Consider a particle moving in the Riemannian space with metric $g_{i j}$ under the influence of the "time" independent potential $V\left(x^{k}\right)$, where by "time" it is understood the affine parameter along the trajectory. The Lagrangian of motion is

$$
L=\frac{1}{2} g_{i j} \dot{x}^{i} \dot{x}^{j}-V\left(x^{k}\right)
$$

A vector field $\mathbf{X}=\xi\left(t, x^{k}\right) \partial_{t}+\eta^{i}\left(t, x^{k}\right) \partial_{x^{i}}$ is a Noether Symmetry of the Lagrangian if condition

$$
\mathbf{X}^{[1]} L+\frac{d \xi}{d t} L=\frac{d f}{d t}
$$


is satisfied, where $\mathbf{X}^{[1]}=\xi\left(t, x^{k}\right) \partial_{t}+\eta^{i}\left(t, x^{k}\right) \partial_{x^{i}}+\left(\frac{d \eta^{i}}{d t}-\dot{x}^{i} \frac{d \xi}{d t}\right) \partial_{\dot{x}^{i}}$ is the first prolongation of $\mathbf{X}$ and $f\left(x^{i}, t\right)$. We compute $\mathbf{X}^{[1]} L$ and noting that the resulting equation is an identity in $\dot{x}^{k}$, we set the coefficient of each power of $\dot{x}^{k}$ equal to zero. We find the following conditions for a Noether symmetry to be admitted by the Lagrangian (44)

$$
\begin{aligned}
V_{, k} \eta^{k}+V \xi_{, t} & =-f_{, t} \\
\eta_{, t}^{i} g_{i j}-\xi_{, j} V & =f_{, j} \\
L_{\eta} g_{i j} & =2\left(\frac{1}{2} \xi_{, t}\right) g_{i j} \\
\xi_{, k} & =0 .
\end{aligned}
$$

Equation (49) implies $\xi=\xi(t)$ and reduces the system as follows

$$
\begin{aligned}
L_{\eta} g_{i j} & =2\left(\frac{1}{2} \xi_{, t}\right) g_{i j} \\
V_{, k} \eta^{k}+V \xi_{, t} & =-f_{, t} \\
\eta_{i, t} & =f_{, i} .
\end{aligned}
$$

Equation (501) implies that $\eta^{i}$ is a conformal Killing vector of the metric provided $\xi_{, t} \neq 0$. Because $g_{i j}$ is independent of $t$ and $\xi=\xi(t)$ the $\eta^{i}$ must be is a HV of the metric. This means that $\eta^{i}(t, x)=T(t) Y^{i}\left(x^{j}\right)$ where $Y^{i}$ is a HV. If $\xi_{, t}=0$ then $\eta^{i}$ is a Killing vector of the metric. Equations (51), (52) are the constraint conditions, which the Noether symmetry and the potential must satisfy for former to be admitted.

We state the solution of the system of equations (50) - (52) as Theorem 2

Theorem 2 The Lagrangian:

$$
L=\frac{1}{2} g_{i j} \dot{x}^{i} \dot{x}^{j}-V\left(x^{k}\right)
$$

of an autonomous conservative dynamical system, moving in a Riemannian space with metric $g_{i j}$, has two sets of Noether symmetries:

a. The Noether symmetry:

$$
\mathbf{X}=\partial_{t}, f=\text { constant }
$$

which accounts for the autonomous character 3 of the potential and has the Noether Integral:

$$
E=\frac{1}{2} g_{i j} \dot{x}^{i} \dot{x}^{j}+V\left(x^{k}\right)
$$

where $E$ is the Hamiltonian of the system.

b. The Noether symmetries which are generated by the homothetic algebra. We have the following cases. Case A: The KVs and the HV satisfy the condition:

$$
V_{, k} Y^{k}+2 \psi_{Y} V+c_{1}=0
$$

The Noether symmetry vector is

$$
\mathbf{X}=2 \psi_{Y} t \partial_{t}+Y^{i} \partial_{i}, \quad f=c_{1} t
$$

\footnotetext{
${ }^{3}$ Note that autonomous is understood with the meaning "independent of the affine parameter along the trajectory". This parameter need not be the time.
} 
where $T(t)=a_{0} \neq 0$. The corresponding Noether integral is $\left(\psi_{Y}=0\right.$ for a $K V$ and 1 for the $\left.H V\right)$

$$
\phi_{I I}=2 \psi_{Y} t E-g_{i j} Y^{i} \dot{x}^{j}+c_{1} t
$$

Case B: The metric admits the gradient $K V s S_{J}$ and the gradient $H V H$ and the potential satisfies the condition

$$
V_{, k} H^{, k}+2 \psi_{H} V=c_{2} H+d
$$

In this case the Noether symmetry vector and the Noether function are

$$
\mathbf{X}=2 \psi_{Y} \int T(t) d t \partial_{t}+T(t) H^{, i} \partial_{i} \quad, \quad f\left(t, x^{k}\right)=T_{, t} H\left(x^{k}\right)+d \int T d t .
$$

The functions $T(t)$ and $K(t)\left(T_{, t} \neq 0\right)$ are computed from the relations

$$
T_{, t t}=c_{2} T, K_{, t}=d \int T d t+\text { constant }
$$

where $c_{2}$ is a constant. The corresponding Noether Integrals are

$$
\phi_{I I I, J}=2 \psi_{H} \int T(t) d t E-g_{i j} H^{i} \dot{x}^{j}+T_{, t} H+d \int T d t .
$$

\section{The Newtonian dynamical systems with two degrees of freedom which admit Lie symmetries}

In this section we apply Theorem 1 to determine all Newtonian dynamical systems with two degrees of freedom which admit at least one Lie point symmetry. The reason for considering this problem is that a Lie point symmetry leads to first integrals, which can be used in many ways to study a given system of differential equations e.g. to simplify, to determine the integrability of the system etc. Because the Newtonian systems move in $E^{2}$ we need to consider the generators of the special projective algebra of $E^{2}$ and then use the constraint conditions for each case to determine the functional form of the force field $F^{i}$.

We consider Cartesian coordinates so that the metric of the space is:

$$
d s^{2}=d x^{2}+d y^{2} .
$$

The special Projective algebra of $E^{2}$ in Cartesian coordinates consists of the following vector fields (see also Table 2):

Table 3: Collineations of Euclidean space $E^{2}$

\begin{tabular}{|l|l|l|}
\hline Collineation & Gradient & Non-gradient \\
\hline Killing vectors (KV) & $\partial_{x}, \partial_{y}$ & $y \partial_{x}-x \partial_{y}$ \\
\hline Homothetic vector (HV) & $x \partial_{x}+y \partial_{y}$ & \\
\hline Affine Collineation (AC) & $x \partial_{x}, y \partial_{y}, y \partial_{x}, x \partial_{y}$ & \\
\hline Special Projective collineation (SPC) & & $x^{2} \partial_{x}+x y \partial_{y}, x y \partial_{x}+y^{2} \partial_{y}$ \\
\hline
\end{tabular}


We note that the special projective algebra of the two dimensional Lorentz space

$$
d s^{2}=-d x^{2}+d y^{2}
$$

is the same with that of the space $E^{2}$, with the difference that the non gradient Killing vector is replaced with $y \partial_{x}+x \partial_{y}$. We shall use this observation in subsection 7.3 where we study the Lie and Noether symmetries of scalar field cosmologies.

\subsection{The case of non-conservative forces}

We examine first the case where the force $F^{i}$ is non-conservative. In the next subsection we consider the case of conservative forces. In certain cases the results are common to both cases, however for clarity it is better to consider the two cases separately. Finally for economy of space, easy reference and convenience we present the results in the form of tables.

In order to indicate how the results of the tables are obtained we consider case A1 of theorem 1 . The Lie point symmetry vectors for case A1 are given by (27) i.e.

$$
\mathbf{X}=\left(\frac{1}{2} d_{1} a_{1} t+d_{2}\right) \partial_{t}+a_{1} Y^{i} \partial_{i}
$$

where $a_{1}$ and $d_{1}$ are constants and $Y^{i}$ is a vector of the affine algebra of $E^{2}$. The force field must satisfy condition (28) i.e.: $L_{Y} \mathbf{F}+d_{1} \mathbf{F}=0$. Writing $\mathbf{F}=F^{x} \partial_{x}+F^{y} \partial_{y}$ and $\mathbf{Y}=Y^{x} \partial_{x}+Y^{y} \partial_{y}$ we obtain a system of two differential equations involving the unknown quantities $F^{x}, F^{y}$ and the known quantities $Y^{x}, Y^{y}$. For each vector $\mathbf{Y}$ we replace $Y^{x}, Y^{y}$ from Table 3 and solve the system to compute $F^{x}, F^{y}$. For example for the gradient $\mathrm{KV} \partial_{x}$ we have $Y^{x}=1, Y^{y}=0$ and find the solution $F^{x}(x, y)=e^{-d x} f(y), F^{y}(x, y)=e^{-d x} g(y)$ where $d$ is a constant and $f(y), g(y)$ are arbitrary (but $C^{\infty}$ ) functions of their argument. Working similarly we determine the form of the force field for all cases of Theorem 1 The results are given in tables 4 and 5 . 
Table 4: Case A1: The affine algebra

\begin{tabular}{|l|l|l|}
\hline $\mathbf{L i e} \downarrow \quad F^{i} \rightarrow$ & $\mathbf{F}^{x}(x, y) / \mathbf{F}^{\theta}(r, \theta)$ & $\mathbf{F}^{y}(x, y) / \mathbf{F}^{\theta}(r, \theta)$ \\
\hline$\frac{d}{2} t \partial_{t}+\partial_{x}$ & $e^{-d x} f(y)$ & $e^{-d x} g(y)$ \\
\hline$\frac{d}{2} t \partial_{t}+\partial_{y}$ & $e^{-d y} f(x)$ & $e^{-d y} g(x)$ \\
\hline$\frac{d}{2} t \partial_{t}+\left(y \partial_{x}-x \partial_{y}\right)$ & $f(r) e^{-d \theta}$ & $g(r) e^{-d \theta}$ \\
\hline$\frac{d}{2} t \partial_{t}+x \partial_{x}+y \partial_{y}$ & $x^{(1-d)} f\left(\frac{y}{x}\right)$ & $x^{(1-d)} g\left(\frac{y}{x}\right)$ \\
\hline$\frac{d}{2} t \partial_{t}+x \partial_{x}$ & $x^{(1-d)} f(y)$ & $x^{-d} g(y)$ \\
\hline$\frac{d}{2} t \partial_{t}+y \partial_{y}$ & $y^{-d} f(x)$ & $y^{(1-d)} g(x)$ \\
\hline$\frac{d}{2} t \partial_{t}+y \partial_{x}$ & $\left(\frac{x}{y} g(y)+f(y)\right) e^{-d \frac{x}{y}}$ & $g(y) e^{-d \frac{x}{y}}$ \\
\hline$\frac{d}{2} t \partial_{t}+x \partial_{y}$ & $f(x) e^{-d \frac{y}{x}}$ & $\left(\frac{y}{x} f(x)+g(x)\right) e^{-d \frac{y}{x}}$ \\
\hline
\end{tabular}

Table 5: Case A2: $Y^{i}$ is a gradient $\mathrm{KV}$ or $\mathrm{HV}$ and $T_{, t t}=m T$.

\begin{tabular}{|l|l|l|}
\hline Lie $\downarrow \quad V \rightarrow$ & $\mathbf{F}^{x}(x, y)$ & $\mathbf{F}^{y}(x, y)$ \\
\hline$T(t) \partial_{x}$ & $-m x+f(y)$ & $g(y)$ \\
\hline$T(t) \partial_{y}$ & $f(x)$ & $-m y+g(x)$ \\
\hline $2 \int T(t) d t \partial_{t}+T(t)\left(x \partial_{x}+y \partial_{y}\right)$ & $-\frac{m}{4} x+x^{-3} f\left(\frac{y}{x}\right)$ & $-\frac{m}{4} y+y^{-3} g\left(\frac{y}{x}\right)$ \\
\hline
\end{tabular}

Case A3: $Y^{i}$ is a special PC

There is only one dynamical system in this case, which is the forced harmonic oscillator, acted upon the external force $F^{i}=(x+a) \partial_{x}+(y+b) \partial_{y}$. As it can be seen from Table 3 the Lie symmetry algebra is the $s l(4, R)$. This result agrees with that of [24].

Except the above three cases we have to consider the Lie point symmetries generated from linear combinations of the vectors $Y^{i}$. It is found that the only new cases are the ones given given in Table 6 and 7 
Table 6: Case A1: $Y^{i}$ is a linear combination of generators of affine collineations

\begin{tabular}{|l|l|l|}
\hline $\mathbf{L i e} \downarrow \quad F^{i} \rightarrow$ & $\mathbf{F}^{x}(x, y) / \mathbf{F}^{r}(r, \theta)$ & $\mathbf{F}^{y}(x, y) / \mathbf{F}^{\theta}(r, \theta)$ \\
\hline$\frac{d}{2} t \partial_{t}+\partial_{x}+b \partial_{y}$ & $f(y-b x) e^{-d x}$ & $g(y-b x) e^{-d x}$ \\
\hline$\frac{d}{2} t \partial_{t}+(a+x) \partial_{x}+(b+y) \partial_{y}$ & $f\left(\frac{b+y}{a+x}\right)(a+x)^{(1-d)}$ & $g\left(\frac{b+y}{a+x}\right)(a+x)^{(1-d)}$ \\
\hline$\frac{d}{2} t \partial_{t}+(a+x) \partial_{x}+(b+h y) \partial_{y}$ & $f\left(\left(\frac{b}{h}+y\right)(a+b x)^{-\frac{h}{b}}\right)(a+b x)^{1-\frac{d}{b}}$ & $g\left(\left(\frac{b}{h}+y\right)(a+b x)^{-\frac{h}{b}}\right)(a+b x)^{\frac{h-d}{b}}$ \\
\hline$\frac{d}{2} t \partial_{t}+(x+y) \partial_{x}+(x+y) \partial_{y}$ & $\left(\begin{array}{c}f(y-x) x+ \\
+g(y-x)\end{array}\right)(y+x)^{-\frac{d}{2}}$ & $\left(\begin{array}{c}f(y-x) y+ \\
-g(y-x)\end{array}\right)(y+x)^{-\frac{d}{2}}$ \\
\hline$\frac{d}{2} t \partial_{t}+\left(a^{2} x+a y\right) \partial_{x}$ & $\begin{array}{c}a(a x+y)^{-\frac{d}{1+a^{2}} \times} \\
\left(x a^{2} f\left(y-\frac{x}{a}\right)+\right. \\
+(a x+y) \partial_{y}\end{array}$ & $\left.\begin{array}{c}a^{2}(a x+y)^{-\frac{d}{1+a^{2}} \times} \\
\times\left(y-\frac{x}{a}\right)+\end{array}\right)$ \\
\hline$\frac{d}{2} t \partial_{t}+(-a y+x) \partial_{x}+(a x+y) \partial_{y}$ & $f(\theta-a \ln r) r^{1-d}$ & $g(\theta-a \ln r) r^{1-d}$ \\
\hline
\end{tabular}

Table 7: Case A2: $Y^{i}$ is a linear combination of gradient $\mathrm{KV}$ or $\mathrm{HV}$ and $T_{, t t}=m T$.

\begin{tabular}{|l|l|l|}
\hline $\mathbf{L i e} \downarrow F^{i} \rightarrow$ & $\mathbf{F}^{x}(x, y)$ & $\mathbf{F}^{y}(x, y)$ \\
\hline$T(t)\left(\partial_{x}+b \partial_{y}\right)$ & $-m x+f(y-b x)$ & $-m b x+g(y-b x)$ \\
\hline $2 \int T(t) d t \partial_{t}+$ & $-\frac{m}{4}(a+x)+$ & $-\frac{m}{4}(b+y)+$ \\
$+T(t)\left[(a+x) \partial_{x}+(b+y) \partial_{y}\right]$ & $+f\left(\frac{b+y}{a+x}\right)(a+x)^{-3}$ & $+g\left(\frac{b+y}{a+x}\right)(a+x)^{-3}$ \\
\hline
\end{tabular}

\subsection{The case of Hamiltonian systems}

In this subsection we assume $F^{i}$ to be conservative with potential function $V(x, y)$. In this case the results of the previous Tables differentiate. Furthermore according to Theorem 1 we have to consider the cases of Case B. The results of the calculations are given below. The results of the calculations are given in tables 8 and 9 . 
Table 8: Case A1: The affine algebra

\begin{tabular}{|l|l|l|l|}
\hline $\mathbf{L i e} \downarrow \quad V \rightarrow$ & $\mathbf{d}=\mathbf{0}$ & $\mathbf{d} \neq \mathbf{0}$ & $\mathbf{d}=\mathbf{2}$ \\
\hline$\frac{d}{2} t \partial_{t}+\partial_{x}$ & $c_{1} x+f(y)$ & $f(y) e^{-d x}$ & $f(y) e^{-2 x}$ \\
\hline$\frac{d}{2} t \partial_{t}+\partial_{y}$ & $c_{1} y+f(x)$ & $f(x) e^{-d y}$ & $f(x) e^{-2 y}$ \\
\hline$\frac{d}{2} t \partial_{t}+\left(y \partial_{x}-x \partial_{y}\right)$ & $\theta+f(r)$ & $f(r) e^{-d \theta}$ & $f(r) e^{-2 \theta}$ \\
\hline$\frac{d}{2} t \partial_{t}+\left(x \partial_{x}+y \partial_{y}\right)$ & $x^{2} f\left(\frac{y}{x}\right)$ & $x^{2-d} f\left(\frac{y}{x}\right)$ & $c_{1} \ln x+f\left(\frac{y}{x}\right)$ \\
\hline$\frac{d}{2} t \partial_{t}+x \partial_{x}$ & $c_{1} x^{2}+f(y)$ & $\nexists$ & $\nexists$ \\
\hline$\frac{d}{2} t \partial_{t}+y \partial_{y}$ & $c_{1} y^{2}+f(x)$ & $\nexists$ & $\nexists$ \\
\hline$\frac{d}{2} t \partial_{t}+y \partial_{x}$ & $x^{2}+y^{2}+c_{1} x$ & $\nexists$ & $\nexists$ \\
\hline$\frac{d}{2} t \partial_{t}+x \partial_{y}$ & $x^{2}+y^{2}+c_{1} y$ & $\nexists$ & $\nexists$ \\
\hline
\end{tabular}

Table 9: Case A2: $Y^{i}$ is a gradient $\mathrm{KV}$ or $\mathrm{HV}$

\begin{tabular}{|l|l|}
\hline Lie $\downarrow \quad V \rightarrow$ & $\mathbf{T}_{, t t}=\mathbf{m} \mathbf{T}$. \\
\hline$T(t) \partial_{x}$ & $-\frac{m x^{2}}{2}+c_{1} x+f(y)$ \\
\hline$T(t) \partial_{y}$ & $-\frac{m y^{2}}{2}+c_{1} y+f(x)$ \\
\hline $2 \int T(t) d t \partial_{t}+T(t)\left(x \partial_{x}+y \partial_{y}\right)$ & $-\frac{m}{8}\left(x^{2}+y^{2}\right)+\frac{1}{x^{2}} f\left(\frac{y}{x}\right)$ \\
\hline
\end{tabular}

The linear combinations give the following new cases listed in tables 10,11 and 12 
Table 10: Case A1: $Y^{i}$ is a linear combination generators of ACs

\begin{tabular}{|l|l|l|}
\hline Lie $\downarrow \quad V \rightarrow$ & $\mathbf{d}=\mathbf{0}$ & $\mathbf{d} \neq \mathbf{0}$ \\
\hline$\frac{d}{2} t \partial_{t}+a \partial_{x}+b \partial_{y}$ & $f(a y-b x)$ & {$\left[c_{1}+f(a y-b x)\right] e^{-d \frac{x}{a}}$} \\
\hline$\frac{d}{2} t \partial_{t}+(a+x) \partial_{x}+(b+y) \partial_{y}$ & $f\left(\frac{b+y}{a+x}\right)(a+x)^{2}$ & $f\left(\frac{b+y}{a+x}\right)(a+x)^{(2-d)}$ \\
\hline$\frac{d}{2} t \partial_{t}+(x+y) \partial_{x}+(x+y) \partial_{y}$ & $f(y-x)+c_{1}(x+y)^{2}$ & $(x+y)^{\left(2-\frac{d}{2}\right)}$ \\
\hline$\frac{d}{2} t \partial_{t}+\left(a^{2} x+a y\right) \partial_{x}+(a x+y) \partial_{y}$ & $c_{1}\left(x^{2}+y^{2}\right)+f(a y-x)$ & $c_{1}(a x+y)\left(2-\frac{d}{1+a^{2}}\right)$ \\
\hline$\frac{d}{2} t \partial_{t}+(-a y+x) \partial_{x}+(a x+y) \partial_{y}$ & $f(\theta-a \ln r) r^{2}$ & $f(\theta-a \ln r) r^{2-d}$ \\
\hline
\end{tabular}

Table 11: Case A1 (continuation of Table 10)

\begin{tabular}{|l|l|l|}
\hline $\mathbf{L i e} \downarrow \quad V \rightarrow$ & $\mathbf{d}=\mathbf{2}$ & $\mathbf{d}=\mathbf{1}$ \\
\hline$\frac{d}{2} t \partial_{t}+\partial_{x}+b \partial_{y}$ & {$\left[c_{1}+f(y-b x)\right] e^{-2 \frac{x}{a}}$} & {$\left[c_{1}+f(y-b x)\right] e^{-\frac{x}{a}}$} \\
\hline$\frac{d}{2} t \partial_{t}+(a+x) \partial_{x}+(b+y) \partial_{y}$ & $f\left(\frac{b+y}{a+x}\right)+c_{1} \ln (a+x)$ & $f\left(\frac{b+y}{a+x}\right)(a+x)$ \\
\hline$\frac{d}{2} t \partial_{t}+(x+y) \partial_{x}+(x+y) \partial_{y}$ & $(x+y)$ & $(x+y)^{\frac{3}{2}}$ \\
\hline$\frac{d}{2} t \partial_{t}+\left(a^{2} x+a y\right) \partial_{x}+(a x+y) \partial_{y}$ & $\ln (a x+y) \quad\left(d=2\left(1+a^{2}\right)\right)$ & $(a x+y)^{\left(\frac{1+2 a^{2}}{1+a^{2}}\right)}$ \\
\hline$\frac{d}{2} t \partial_{t}+(-a y+x) \partial_{x}+(a x+y) \partial_{y}$ & $c_{1} \ln r+f(\theta-a \ln r)$ & $c_{1} r+f(\theta-a \ln r) r$ \\
\hline
\end{tabular}

Table 12: Case A2 $Y^{i}$ is a linear combination generators of the gradient HVs

\begin{tabular}{|l|l|}
\hline Lie $\downarrow V \rightarrow$ & $\mathbf{T}_{, t t}=\mathbf{m} \mathbf{T}$ \\
\hline$T(t)\left(a \partial_{x}+b \partial_{y}\right)$ & $-\frac{m}{2}\left(x^{2}+y^{2}\right)+c_{1} x+f(a y-b x)$ \\
\hline $2 \int T(t) d t \partial_{t}+T(t)\left[(a+x) \partial_{x}+(b+y) \partial_{y}\right]$ & $-\frac{m}{8}\left(x^{2}+y^{2}+2 a x+2 b y\right)+(a+x)^{-2} f\left(\frac{b+y}{a+x}\right)$ \\
\hline
\end{tabular}

As it was stated in section 1 the determination of all two dimensional potentials which admit a Lie point symmetry has been addressed previously in [8] and [9]. Our results contain the results of both these papers and additionally some cases missing, mainly in the linear combinations of the HV with the KVs. Obviously the derivation discussed above is systematic and can be generalized to higher dimensions in a straightforward manner. However the possible symmetries increase dramatically so this task can only be considered elsewhere.

\section{The Newtonian dynamical systems with two degrees of freedom which admit Noether symmetries}

Noether symmetries are associated with a Lagrangian. Therefore we consider only the case in which the force $F^{i}$ is conservative. Furthermore, Noether symmetries are special Lie point symmetries, hence we look into the two 
dimensional potentials which admit a Lie point symmetry. These potentials were determined in the previous section. We apply Theorem 2 to these potentials and select the potentials which admit a Noether symmetry. The calculations are similar to the ones for the Lie point symmetries and are omitted. The results are listed in tables $13,14,15$ and 16 .

Table 13: Case A. $Y^{i}$ is a HV

\begin{tabular}{|l|l|}
\hline Noether Symmetry & $\mathbf{V}(x, y)$ \\
\hline$\partial_{x}$ & $c x+f(y)$ \\
\hline$\partial_{y}$ & $c y+f(x)$ \\
\hline$y \partial_{x}-x \partial_{y}$ & $c \theta+f(r)$ \\
\hline $2 t \partial_{t}+x \partial_{x}+y \partial_{y}$ & $x^{-2} f\left(\frac{y}{x}\right)$ \\
\hline
\end{tabular}

Table 14: Case A. $Y^{i}$ is a linear combination of HVs

\begin{tabular}{|l|l|}
\hline Noether Symmetries & $\mathbf{V}(x, y)$ \\
\hline$\partial_{x}+b \partial_{y}$ & $f(y-b x)-c x$ \\
\hline$(a+y) \partial_{x}+(b-x) \partial_{y}$ & $f\left(\frac{1}{2}\left(x^{2}+y^{2}\right)+a y-b x\right)$ \\
\hline $2 t \partial_{t}+(x+a y) \partial_{x}+(y-a x) \partial_{y}$ & $r^{-2} f(\theta-a \ln r)$ \\
\hline $2 t \partial_{t}+(a+x) \partial_{x}+(b+y) \partial_{y}$ & $f\left(\frac{b+x}{a+x}\right)(a+x)^{-2}-c(a+x)^{-2}\left(\frac{1}{2} x^{2}+a x\right)$ \\
\hline
\end{tabular}

Table 15: Case B. $Y^{i}$ is a gradient HV

\begin{tabular}{|l|l|}
\hline Noether $\downarrow V \rightarrow$ & $\mathbf{T}_{, t t}=\mathbf{m} \mathbf{T}$ \\
\hline$T(t) \partial_{x}$ & $f(y)-c x-\frac{m}{2} x^{2}$ \\
\hline$T(t) \partial_{y}$ & $f(x)-c y-\frac{m}{2} y^{2}$ \\
\hline $2 \int T(t) d t \partial_{t}+T(t)\left(x \partial_{x}+y \partial_{y}\right)$ & $x^{-2} f\left(\frac{y}{x}\right)-\frac{m}{8}\left(x^{2}+y^{2}\right)$ \\
\hline
\end{tabular}

Table 16: Case B. $Y^{i}$ is a linear combination of gradient HVs

\begin{tabular}{|l|l|}
\hline Noether $\downarrow \quad V \rightarrow$ & $\mathbf{T}_{, t t}=\mathbf{m} \mathbf{T}$ \\
\hline$T(t) \partial_{x}+b T(t) \partial_{y}$ & $-\frac{m}{2}\left(x^{2}+y^{2}\right)-\frac{m}{2}(y-b x)^{2}+f(y-b x)-c x$ \\
\hline $2 \int T(t) d t \partial_{t}+T(t)\left((a+x) \partial_{x}+(b+y) \partial_{y}\right)$ & $f\left(\frac{b+x}{a+x}\right)(a+x)^{-2}-\frac{c}{2}(a+x)^{-2} x(x+2 a)$ \\
& $-\frac{x m(x+2 a)}{8(a+x)^{4}}\left\{\begin{array}{c}\left((a+x)^{2}+a^{2}\right) y(y+2 b)+ \\
+x(x+2 a)(b+(a+x))(-b+(a+x))\end{array}\right.$ \\
\hline
\end{tabular}




\section{Applications}

In this section we demonstrate the application of the results of sections 5 and 6 in three cases. The first case is the Kepler-Ermakov system, which (in general) is not a conservative dynamical system, the second is the Hènon - Heiles type potential and the third is the scalar field cosmology.

\subsection{The Lie Symmetries of the Kepler-Ermakov system.}

The Ermakov systems are time dependent dynamical systems, which contain an arbitrary function of time (the frequency function) and two arbitrary homogeneous functions of dynamical variables. A central feature of Ermakov systems is their property of always having a first integral. The Kepler-Ermakov system is an autonomous Ermakov system defined by the equations [15.

$$
\begin{aligned}
& \ddot{x}+\frac{x}{r^{3}} H(x, y)-\frac{1}{x^{3}} f\left(\frac{y}{x}\right)=0 \\
& \ddot{y}+\frac{y}{r^{3}} H(x, y)-\frac{1}{y^{3}} g\left(\frac{y}{x}\right)=0
\end{aligned}
$$

where $H, f, g$ are arbitrary functions. In [10] it has been shown that this system admits Lie point symmetries for certain forms of the function $H(x, y)$. Furthermore it has been shown that for special classes of these equations there exists a Lagrangian (see also [1]).

In the following we demonstrate the use of our results by finding the Lie symmetries simply by reading the entries of the proper tables. Looking at the tables we find that equations (65), (66) admit a Lie point symmetry for the following two cases.

Case 1.

When $H(x, y)=\frac{h\left(\frac{y}{x}\right)}{x}$. Then line 4 of Table 4 applies for $d=4$. Also line 3 of Table 5 for $m=0$. Therefore the Lie point symmetries are:

$$
X=\left(c_{1}+c_{2} 2 t+c_{3} t^{2}\right) \partial_{t}+\left(c_{2} x+c_{3} t x\right) \partial_{x}+\left(c_{2} y+c_{3} t y\right) \partial_{y}
$$

Case 2.

When $H(x, y)=\omega^{2} r^{3}+\frac{h\left(\frac{y}{x}\right)}{x}$ where $m=-4 \omega^{2}$ and $m \neq 0$. In this case line 3 of Table 5 for $m \neq 0$ applies and the Lie point symmetry generator is

$$
X=\left(c_{1}-\frac{c_{2}}{\omega} \cos (2 \omega t)+\frac{c_{3}}{\omega} \sin (2 \omega t)\right) \partial_{t}+\left(c_{2} \sin (2 \omega t)+c_{3} \cos (2 \omega t)\right) x \partial_{x}+\left(c_{2} \sin (2 \omega t)+c_{3} \cos (2 \omega t)\right) y \partial_{y} .
$$

These symmetries coincide with the ones found in [10]. We note that in both cases the Lie symmetry vectors come from the $\mathrm{HV} x \partial_{x}+y \partial_{y}$ of the Euclidean metric.

In a subsequent publication [11] it was shown that the Lagrangian considered in [10] was incorrect and that the correct Lagrangian is:

$$
L=\frac{1}{2}\left(\dot{r}^{2}+r^{2} \dot{\theta}^{2}\right)-\frac{1}{2} \omega^{2} r^{2}-\frac{\mu}{2 r^{2}}-\frac{C(\theta)}{2 r^{2}}
$$

where $C(\theta)=\sec ^{2} \theta f(\tan \theta)+\csc ^{2} \theta g(\tan \theta)$ and the functions $f, g$ satisfy two compatibility conditions (see equation (5.2) of [11]).

In order to find the Noether symmetries of this Lagrangian we note that for every function $C(\theta)$ the potential is of the form $V(r, \theta)=\frac{m}{2}\left(x^{2}+y^{2}\right)+x^{-2} V\left(\frac{y}{x}\right)$. This means that line 4 of Table 13 and line 3 of Table 15 
with $\omega=0, m=0$ apply. It follows that the Lie symmetries are also Noether symmetries and that the Noether Integrals (in addition to the Hamiltonian $E$ ) corresponding the these Noether symmetries are

$$
\begin{aligned}
& I_{1}=2 t E-r \dot{r} \\
& I_{2}=t^{2} E-t r \dot{r}+\frac{1}{2} r^{2} .
\end{aligned}
$$

In total we have three Noether integral 4 . Since we do not look for generalized symmetries, we do not expect to find the Ermakov - Lewis invariant [12].

\subsection{On the Hènon - Heiles potential}

The Hènon - Heiles potential

$$
V(x, y)=\frac{1}{2}\left(x^{2}+y^{2}\right)+x^{2} y-\frac{1}{3} y^{2}
$$

has been used as a model for the galactic cluster. Computer analysis has suggested that for sufficiently small values of the energy, there exists a first integral independent of energy. In [13] it is proposed to study if there exists a Lie point symmetry of the potential which could justify such a first integral. Working in a slightly more general scenario, in [13] are considered potentials of the form

$$
V(x, y)=\frac{1}{2}\left(x^{2}+y^{2}\right)+A x^{3}+B x^{2} y+C x y^{2}+D y^{3}
$$

where $A, B, C, D$ are real parameters. The Hènon - Heiles potential is the special case for $A=C=0, B=$ $1, D=-\frac{1}{3}$.

Using standard Lie analysis in [13] it is shown that only the potentials $V_{1}(x, y)=\frac{1}{2}\left(x^{2}+y^{2}\right)+x^{3}, V_{2}(x, y)=$ $\frac{1}{2}\left(x^{2}+y^{2}\right)+y^{3}, \quad V_{3}(x, y)=\frac{1}{2}\left(x^{2}+y^{2}\right) \pm(a y \pm x)^{3}, \quad V_{4}(x, y)=\frac{1}{2}\left(x^{2}+y^{2}\right) \pm(a y \mp x)^{3}$ admit Lie point symmetries, hence the Hènon - Heiles potential does not admit a Lie symmetry and the existence of a first integral it is not justified. We apply the results of sections 5 , 6 to give the Lie point symmetries and the Noether quantities of these potentials, simply by reading the relevant tables.

The potential $V_{1}(x, y)$ is of the form $c y^{2}+f(x)$ and belongs to the types:

\begin{tabular}{|l|l|l|l|l|}
\hline Table & Line & Parameters & Lie & Type of vector \\
\hline 8 & 6 & $d=0, c_{1}=\frac{1}{2}, f(x)=\frac{1}{2} x^{2}+x^{3}$ & $y \partial_{y}$ & Affine \\
\hline 9 & 2 & $m=-1, c_{1}=0, f(x)=\frac{1}{2} x^{2}+x^{3}$ & $(\cos t$ or $\sin t) \partial_{y}$ & gradient KV \\
\hline
\end{tabular}

Therefore the Lie symmetries admitted by this potential are:

$$
X=c_{0} \partial_{t}+c_{1} \sin t \partial_{y}+c_{2} \cos t \partial_{y}+c_{3} y \partial_{y} .
$$

\footnotetext{
${ }^{4}$ When $\omega \neq 0$ only line 3 of Table 15 for $m=-4 \omega^{2}$ applies and the Lie symmetry is also Noether symmetry with Noether integrals

$$
\begin{aligned}
& I_{1}^{\prime}=-\frac{1}{\omega} \cos (2 \omega t) E-\sin (2 \omega t) r \dot{r}+\omega \cos (2 \omega t) r^{2} \\
& I_{2}^{\prime}=\frac{1}{\omega} \sin (2 \omega t) E-\cos (2 \omega t) r \dot{r}-\omega \sin (2 \omega t) r^{2}
\end{aligned}
$$


We note that the Lie symmetry $y \partial_{y}$, which is due to the Affine collineation, has not been found in [13].

The potential $V_{2}(x, y)$ is obtained by $V_{1}(x, y)$ with $x, y$ interchanged. Therefore the Lie symmetries admitted by the potential $V_{2}(x, y)$ are:

$$
X=c_{0} \partial_{t}+c_{1} \sin t \partial_{x}+c_{2} \cos t \partial_{x}+c_{3} x \partial_{x}
$$

and again in 13 the Lie point symmetry $y \partial_{y}$ is missing.

The potential $V_{3}(x, y)$ is of the form $\frac{1}{2}\left(x^{2}+y^{2}\right)+f(x-a y)$ and belongs to the types:

\begin{tabular}{|l|l|l|l|l|}
\hline Table & Line & Parameters & Lie & Type of vector \\
\hline 12 & 1 & $m=-1, c_{1}=0, b= \pm 1, \neq 0$, & $(\cos t$ or $\sin t)\left(a \partial_{x} \pm \partial_{y}\right)$ & gradient KV \\
\hline 10 & 5 & $d=0, c_{1}=\frac{1}{2}, f= \pm(a y \pm x)^{3}$ & $(a x+y)\left(a \partial_{x}+\partial_{y}\right)$ & two gradient KVs \\
\hline
\end{tabular}

Therefore its Lie point symmetries are:

$$
X=c_{0} \partial_{t}+\left(c_{1} \cos t+c_{2} \sin t\right)\left(a \partial_{x} \pm \partial_{y}\right)+c_{3}(a x+y)\left(a \partial_{x}+\partial_{y}\right) .
$$

The potential $V_{4}(x, y)$ is of the same form as $V_{3}(x, y)$ with $x, y$ interchanged. Therefore the Lie point symmetries are:

$$
X=c_{0} \partial_{t}+a\left(c_{1} \cos t+c_{2} \sin t\right)\left(a \partial_{x} \mp \partial_{y}\right)+c_{3}(a x+y)\left(a \partial_{x}+\partial_{y}\right)
$$

We observe that in all four cases the Lie symmetries depend on four free parameters (the $\left.c_{0}, c_{1}, c_{2}, c_{3}\right)$. The parameter $c_{0}$ determines the vector $c_{0} \partial_{t}$ and the rest $c_{1}, c_{2}, c_{3}$ the $x-y$ part of the symmetry generators.

The Lie point symmetries which are possibly Noether symmetries are the ones generated by the KVs. We check that the Lie point symmetries which are due to the gradient KVs (with $m \neq 0$ ) are Noether Symmetries of the potentials (plus the $\partial_{t}$ whose Noether integral is the Hamiltonian). The Noether integrals and the Noether functions corresponding to each of these symmetries are given in Table 17.

Table 17: Noether symmetries admitted by the potentials $V_{1}, V_{2}, V_{3}, V_{4}$

\begin{tabular}{|l|l|l|}
\hline $\mathbf{V}(x, y)$ & Noether Symmetry & Noether Integral \\
\hline \multirow{2}{*}{$\frac{1}{2}\left(x^{2}+y^{2}\right)+x^{3}$} & $\sin t \partial_{y}$ & $\dot{y} \sin t-y \cos t$ \\
\cline { 2 - 3 } & $\cos t \partial_{y}$ & $\dot{y} \cos t+y \sin t$ \\
\hline \multirow{2}{*}{$\frac{1}{2}\left(x^{2}+y^{2}\right)+y^{3}$} & $\sin t \partial_{x}$ & $\dot{x} \sin t-x \cos t$ \\
\cline { 2 - 3 } & $\cos t \partial_{x}$ & $\dot{x} \cos t+x \sin t$ \\
\hline \multirow{2}{*}{$\frac{1}{2}\left(x^{2}+y^{2}\right) \pm(a y \pm x)^{3}$} & $\sin t\left(\mp a \partial_{x}+\partial_{y}\right)$ & $(\mp a \dot{x}+\dot{y}) \sin t-(\mp a x+y) \cos t$ \\
\cline { 2 - 3 } & $\cos t\left(\mp a \partial_{x}+\partial_{y}\right)$ & $(\mp a \dot{x}+\dot{y}) \cos t+(\mp a x+y) \sin t$ \\
\hline \multirow{2}{*}{$\frac{1}{2}\left(x^{2}+y^{2}\right) \pm(a y \mp x)^{3}$} & $\sin t\left( \pm a \partial_{x}+\partial_{y}\right)$ & $( \pm a \dot{x}+\dot{y}) \sin t-( \pm a x+y) \cos t$ \\
\cline { 2 - 3 } & $\cos t\left( \pm a \partial_{x}+\partial_{y}\right)$ & $( \pm a \dot{x}+\dot{y}) \cos t+( \pm a x+y) \sin t$ \\
\hline
\end{tabular}

These results coincide with those of [13, 14]. 


\subsection{Scalar field cosmology}

Scalar field cosmological models are used extensively in the study of dynamics of the inflationary universe [16] and the dynamics of unified UDM [17, 18. In these models one normally considers a Friedmann Robertson Walker (FRW) space time and a scalar field with potential $V(\phi)$ minimally coupled to the gravitational field. The form of the scalar field potential is taken at will, without a deeper physical or geometrical hypothesis. In the following we propose that the form of the potential should be fixed by the requirement that the Lagrangian of the equations of "motion" admits a second Noether symmetry (besides the trivial $\partial_{t}$ ). In this case one has two Noether symmetries, hence two Noether integrals, and the dynamical system will be integrable.

The gravitational field equation 5 of the FRW space time containing a scalar field $\phi$ only are (see e.g. [18, [19]):

$$
\begin{aligned}
3\left(H^{2}+\frac{k}{a^{2}}\right) & =\frac{1}{2} \dot{\phi}^{2}+V(\phi) \\
2 \frac{\ddot{a}}{a}+H^{2}+\frac{k}{a^{2}} & =-\frac{1}{2} \dot{\phi}^{2}+V(\phi)
\end{aligned}
$$

and are supplied by the field equation (known as the Klein Gordon equation) for $\phi$ :

$$
\ddot{\phi}+3 H \phi+V^{\prime}(\phi)=0
$$

Here $H=\frac{\dot{a}}{a}$ is the Hubble parameter, $k=0, \pm 1$ is the curvature of the 3 -d space and an overdot (respectively prime) indicates derivation with respect to time (respectively $\phi$ ). The scalar field is assumed to inherit the symmetries of the space. Equation (74) is equivalent to the matter conservation equation

$$
\dot{\mu}=-3(\mu+p) H
$$

where $\mu$ is the matter energy density of the FRW spacetime. This is trivially satisfied, because we have assumed that space time contains a scalar field only (hence $\mu=0$ ). We conclude that the scalar field cosmological model (for all potentials $V(\phi)$ !) can be considered as a dynamical system with two degrees of freedom $a, \phi$ defined by the equations (75), (76) or, equivalently, by the Lagrangian:

$$
L=3 a \dot{a}^{2}-a^{3} \frac{\dot{\phi}^{2}}{2}+a^{3} V(\phi)-3 k a .
$$

We apply Theorem 2 to determine the forms of the potential $V(\phi)$ for which the Lagrangian (78) admits Noether symmetries. To do that we write the Lagrangian in the form $L=T-U$, where:

$$
\begin{aligned}
T & =3 a \dot{a}^{2}-a^{3} \frac{\dot{\phi}^{2}}{2} \\
U & =-a^{3} V(\phi)+3 k a .
\end{aligned}
$$

In the phase space of $a, \phi$ the kinetic energy defines the metric:

$$
d s^{2}=6 a d a^{2}-a^{3} d \phi^{2}
$$

\footnotetext{
${ }^{5}$ We set $8 \pi G=c=1$.

${ }^{6}$ That is the scalar field satisfies the condition $\mathcal{L}_{\xi} \phi=0$ for all Killing vectors $\xi^{a}$ of the metric. Because the 3-spaces are maximally symmetric this implies that $\phi=\phi(t)$.
} 
making this space an Einstein space. We compute the Ricci scalar $R=0$, hence the space is a two dimensional Lorentzian flat space. Applying the transformation:

$$
x=\sqrt{3} a^{3 / 2} \sinh \left(\sqrt{\frac{3}{2}} \phi\right), y=\sqrt{3} a^{3 / 2} \cosh \left(\sqrt{\frac{3}{2}} \phi\right)
$$

the metric is written in its canonical form and in the canonical coordinates $x, y$ the Lagrangian becomes:

$$
L=\frac{1}{2}\left(\dot{y}^{2}-\dot{x}^{2}\right)+\frac{1}{2}\left(y^{2}-x^{2}\right) V\left(\frac{y}{x}\right)-k\left(y^{2}-x^{2}\right)^{\frac{1}{3}}
$$

where we have absorbed the constants into $V\left(\frac{y}{x}\right)$.

We determine the Lie and the Noether symmetries of this Lagrangian for the cases $k=0, k \neq 0$ assuming that $V(\phi) \neq$ constant.

Case 1: $k=0$

Lie symmetries

From Table 8 , Line 4 with $d=0$ we find that the Lie symmetries are

$$
X_{A}=c_{1} \partial_{t}+c_{2}\left(x \partial_{x}+y \partial_{y}\right)
$$

where $V\left(\frac{y}{x}\right)$ is an arbitrary function of its argument.

From Table 10, line 5 with $d \neq 0$ we find the Lie symmetry:

$$
X_{B}=\left(c_{1}+c_{3} 2 t\right) \partial_{t}+c_{2}\left(x \partial_{x}+y \partial_{y}\right)+c_{3} \frac{4}{d}\left(y \partial_{x}+x \partial_{y}\right)
$$

and $V\left(\frac{y}{x}\right)=e^{-d \arctan \frac{y}{x}}$. In the special case $d= \pm 2$ then the system admits the additional Lie symmetry $\partial_{x} \pm \partial_{y}$

For $V\left(\frac{y}{x}\right)=\frac{\omega_{1}}{2} \frac{x^{2}}{x^{2}-y^{2}}-\frac{\omega_{2}}{2} \frac{y^{2}}{x^{2}-y^{2}}$ where $\omega_{1}, \omega_{2}$ are constants and $\omega_{1} \neq \omega_{2}$, the Lagrangian (82) describes the anisotropic oscillator.

From Table 8, lines 5 and 6 and Table 9 lines 1,2 we read the generic Lie symmetry:

$$
X_{C}=c_{1} \partial_{t}+\left(c_{2} \sin \left(\sqrt{\omega_{1}} t\right)+c_{3} \cos \left(\sqrt{\omega_{1}} t\right)+c_{4} x\right) \partial_{x}+\left(c_{5} \sin \left(\sqrt{\omega_{2}} t\right)+c_{6} \cos \left(\sqrt{\omega_{2}} t\right)+c_{7} y\right) \partial_{y} .
$$

For $V\left(\frac{y}{x}\right)=\frac{\omega_{1}}{2} \frac{x^{2}}{x^{2}-y^{2}}\left(\right.$ i.e. $\left.\omega_{2}=0\right)$ we find the Lie symmetry:

$$
X_{C_{1}}=c_{1} \partial_{t}+\left(c_{2} \sin \left(\sqrt{\omega_{1}} t\right)+c_{3} \cos \left(\sqrt{\omega_{1}} t\right)+c_{4} x\right) \partial_{x}+\left(c_{5}+c_{6} t+c_{7} y\right) \partial_{y} .
$$

For $V\left(\frac{y}{x}\right)=-\frac{\omega_{2}}{2} \frac{y^{2}}{x^{2}-y^{2}} \quad\left(\right.$ i.e. $\left.\omega_{1}=0\right)$ we find the Lie symmetry:

$$
X_{C_{2}}=c_{1} \partial_{t}+\left(c_{2}+c_{3} t+c_{4} x\right) \partial_{x}+\left(c_{5} \sin \left(\sqrt{\omega_{2}} t\right)+c_{6} \cos \left(\sqrt{\omega_{2}} t\right)+c_{7} y\right) \partial_{y} .
$$

Noether symmetries

For $V\left(\frac{y}{x}\right)=$ arbitrary there is only the standard Noether symmetry $\partial_{t}$, with Noether integral the Hamiltonian E.

For $V\left(\frac{y}{x}\right)=e^{-d \arctan \frac{y}{x}}$ we have the extra Noether symmetry from Table 14, line 3 :

$$
2 t \partial_{t}+\left(x \partial_{x}+\frac{4}{d} y \partial_{x}\right)+\left(y \partial_{y}+\frac{4}{d} x \partial_{y}\right)
$$


with Noether integral

$$
\phi=2 t E+\left(x+\frac{4}{d} y\right) \dot{x}-\left(y+\frac{4}{d} x\right) \dot{y} .
$$

For the value $d= \pm 2$, we have the extra Noether symmetry $\partial_{x} \pm \partial_{y}$ whose Noether Integral is $\phi_{d=2}=\dot{x} \mp \dot{y}$ For $V\left(\frac{y}{x}\right)=\frac{\omega_{1}}{2} \frac{x^{2}}{x^{2}-y^{2}}-\frac{\omega_{2}}{2} \frac{y^{2}}{x^{2}-y^{2}}$ we have the extra Noether symmetries from Table 15 lines 1 and 2

$$
X_{N}=\left(n_{2} \sin \left(\sqrt{\omega_{1}} t\right)+n_{3} \cos \left(\sqrt{\omega_{1}} t\right)\right) \partial_{x}+\left(n_{4} \sin \left(\sqrt{\omega_{2}} t\right)+n_{5} \cos \left(\sqrt{\omega_{2}} t\right)\right) \partial_{y}
$$

with Noether integrals

$$
\begin{aligned}
& I_{n_{2}}=\sin \left(\sqrt{\omega_{1}} t\right) \dot{x}-\sqrt{\omega_{1}} \cos \left(\sqrt{\omega_{1}} t\right) x \\
& I_{n_{3}}=\cos \left(\sqrt{\omega_{1}} t\right) \dot{x}+\sqrt{\omega_{1}} \sin \left(\sqrt{\omega_{1}} t\right) x \\
& I_{n_{4}}=\sin \left(\sqrt{\omega_{2}} t\right) \dot{y}-\sqrt{\omega_{2}} \cos \left(\sqrt{\omega_{2}} t\right) y \\
& I_{n_{5}}=\cos \left(\sqrt{\omega_{2}} t\right) \dot{y}+\sqrt{\omega_{2}} \sin \left(\sqrt{\omega_{2}} t\right) y
\end{aligned}
$$

For $V\left(\frac{y}{x}\right)=\frac{\omega_{1}}{2} \frac{x^{2}}{x^{2}-y^{2}}$ we have, from the same table and lines, the extra Noether symmetries

$$
X_{N_{1}}=\left(n_{H O_{1}} \sin \left(\sqrt{\omega_{1}} t\right)+n_{H O_{2}} \cos \left(\sqrt{\omega_{1}} t\right)\right) \partial_{x}+\left(n_{F_{1}}+n_{F_{2}} t\right) \partial_{y}
$$

with Noether integrals

$$
\begin{aligned}
I_{H O_{1}} & =\sin \left(\sqrt{\omega_{1}} t\right) \dot{x}-\sqrt{\omega_{1}} \cos \left(\sqrt{\omega_{1}} t\right) x \\
I_{H O_{1}} & =\cos \left(\sqrt{\omega_{1}} t\right) \dot{x}+\sqrt{\omega_{1}} \sin \left(\sqrt{\omega_{1}} t\right) x \\
I_{F_{1}} & =\dot{y}, I_{F_{2}}=t \dot{y}-y .
\end{aligned}
$$

For $V\left(\frac{y}{x}\right)=-\frac{\omega_{2}}{2} \frac{y^{2}}{x^{2}-y^{2}}$ we have, from the same table and lines, the extra Noether symmetries

$$
X_{N_{2}}=\left(n_{F_{1}}+n_{F_{2}} t\right) \partial_{x}+\left(n_{H O_{1}} \sin \left(\sqrt{\omega_{2}} t\right)+n_{H O_{2}} \cos \left(\sqrt{\omega_{2}} t\right)\right) \partial_{y}
$$

with Noether integrals

$$
\begin{aligned}
I_{H O_{1}} & =\sin \left(\sqrt{\omega_{2}} t\right) \dot{y}-\sqrt{\omega_{2}} \cos \left(\sqrt{\omega_{2}} t\right) y \\
I_{H O_{1}} & =\cos \left(\sqrt{\omega_{2}} t\right) \dot{y}+\sqrt{\omega_{2}} \sin \left(\sqrt{\omega_{2}} t\right) y \\
I_{F_{1}} & =\dot{x}, I_{F_{2}}=t \dot{x}-x .
\end{aligned}
$$

Case 2: $k \neq 0$

Lie symmetries

For the arbitrary potential $V\left(\frac{y}{x}\right)$ there is only the trivial Lie symmetry $\partial_{t}$.

For the potential $V\left(\frac{y}{x}\right)=e^{-C \arctan \frac{y}{x}}$ we have from Table 10 , line 5 with $d \neq 0$ the extra Lie symmetry:

$$
\frac{2}{3} t \partial_{t}+\left(x \partial_{x}+y \partial_{y}\right)+\frac{4}{3 C}\left(y \partial_{x}+x \partial_{y}\right)
$$

Noether symmetries

For all potentials only the trivial Noether symmetry $\partial_{t}$ is admitted.

We conclude that in a flat (i.e. $k=0$ ) FRW spacetime with no matter present the scalar field cosmological models which admit Noether symmetries, hence possibly integrable, are the ones defined by the UDM potential $\left(\omega_{1,2}<0\right)[18$ and the exponential potential. Indeed, the analytical solution for the exponential potential has been given in [19]. 


\section{Conclusion}

We have presented two theorems which relate the Lie point symmetries and the Noether symmetries of the second order system (1) with the generators of the special projective algebra and the homothetic algebra of the metric respectively. Therefore if one knows these algebras of a Riemannian space, then one computes the Lie (and the Noether) symmetries of all dynamical systems moving in that space, by simply checking which $Y^{i \prime} s$ satisfy the appropriate constraint conditions with $F^{i}$. We have applied these theorems to classify all two dimensional Newtonian dynamical systems which admit at least one Lie point symmetry, and in the case of conservative forces, all potentials $V(x, y)$ which admit a Lie point symmetry and a Noether symmetry.

We have demonstrated the application of the results in various important cases. We considered the KeplerErmakov system, which is an autonomous, but in general not conservative dynamical system and we determined the classes of this type of systems which admit Lie point and Noether symmetries; we also considered the case of the Hènon Heiles type potentials and determined their Lie point symmetries and their Noether symmetries. These results are compatible and complete previous results in the literature. We continued with scalar field cosmological models and proved that the exponential potential and the UDM potential are the only scalar field potentials in a flat FRW spacetime in the absence matter, which lead to integrable models.

The conclusion from the above is that the symmetries of a space modulate the motion of dynamical systems moving in that space. That is, the space (i.e. the metric) is not a simple substratum in which a dynamical system develops its motion according to an external force (cause), but it is the space itself which, via the special projective algebra, selects those forces which posses Lie point symmetries and Noether symmetries. An equivalent way to state this conclusion is to say that a dynamical system moving in a Riemannian space has conserved quantities, in the standard sense of first integrals and/or Noether integrals, only if the force causing the motion is compatible with the geometry (i.e. metric) of the space.

There are many directions that the present work can be extended. The immediate topic to consider is the classification of all three dimensional Newtonian dynamical systems, conservative and non-conservative, which admit Lie point and Noether symmetries 20]. However initial work has shown, that this topic involves a large number of cases and must be considered separately. Perhaps the next step to be taken is to depart form the easy case of flat spaces and consider motion in curved Riemannian spaces. Following [8], it would also be interesting to use the above results, and study the relation between the Lie point / Noether symmetries and the integrability of second order systems. Of course, a wealth of results will follow if the theorems presented in this paper are generalized to the case of a time dependent potential, a problem on which we are currently involved.

Acknowledgements The authors thank Professor P.G.L. Leach for valuable comments and corrections; also an anonymous referee for the useful remarks and references he kindly provided.

\section{A Appendix}

In this appendix we discuss the relation between the Lie symmetries of the harmonic oscillator and those of a free particle.

We consider first the one dimensional case. 
The equation of motion of the damped, attractive or repulsive, forced, time dependent harmonic oscillator is:

$$
\frac{d^{2} x}{d t^{2}}+\gamma \frac{d x}{d t}+\varepsilon \omega^{2}(t)-f(t)=0
$$

where $\gamma$ is a constant and $\varepsilon=+1$ for the attractive and -1 for the repulsive oscillator.

Theorem 3.4 (p. 961) of [3] states that:

The second order differential equation

$$
\frac{d^{2} x}{d t^{2}}+a(t, x)\left(\frac{d x}{d t}\right)^{3}+b(t, x)\left(\frac{d x}{d t}\right)^{2}+c(t, x) \frac{d x}{d t}+d(t, x)=0
$$

can be reduced by a change of variables $(t, x) \rightarrow(\bar{t}, \bar{x})$ to the equation

$$
\frac{d^{2} \bar{x}}{d \bar{t}^{2}}=0
$$

if and only if the following conditions hold:

$$
\begin{aligned}
& 3 \frac{\partial^{2} d}{\partial x^{2}}-2 \frac{\partial^{2} c}{\partial t \partial x}+3 d \frac{\partial b}{\partial x}+3 b \frac{\partial d}{\partial x}-2 c \frac{\partial c}{\partial x}+\frac{\partial^{2} b}{\partial t^{2}}+c \frac{\partial b}{\partial t}-6 d \frac{\partial a}{\partial t}-3 a \frac{\partial d}{\partial t}=0 \\
& \frac{\partial^{2} c}{\partial x^{2}}-2 \frac{\partial^{2} b}{\partial t \partial x}-b \frac{\partial c}{\partial x}+3 d \frac{\partial a}{\partial x}+6 a \frac{\partial d}{\partial x}+3 \frac{\partial^{2} a}{\partial t^{2}}+2 b \frac{\partial b}{\partial t}-3 c \frac{\partial a}{\partial t}-3 a \frac{\partial c}{\partial t}=0
\end{aligned}
$$

Comparing (83) with (84) we find:

$$
a=b=0, c=\gamma, d=\varepsilon \omega^{2}(t) x-f(t) .
$$

It is easy to show that for these values of $a, b, c, d$ conditions (86), (87) are satisfied, therefore there exists a coordinate transformation which transforms equation (83) to equation (85). Because under a change of variables the Lie symmetry algebra of the equation does not change, we infer that the damped, attractive or repulsive, forced, time dependent harmonic oscillator shares the same Lie symmetry algebra (i.e. the $s l(3, R)$, with the free particle [21, 22, 23].

We continue with the $n$ dimensional case.

The isotropic time dependent $n$ dimensional harmonic oscillator is defined by the equation [24]:

$$
\frac{d^{2} x^{i}}{d t^{2}}+\varepsilon \omega^{2}(t) x^{i}=0
$$

In order to check that (88) is reducible to the free particle equation of motion (i..e. the geodesic equations) we use Theorem 3.2. (p. 960) of [3] which states that:

The differential system

$$
\frac{d^{2} x^{i}}{d t^{2}}+a_{j k}(x, t) \frac{d x^{i}}{d t} \frac{d x^{j}}{d t} \frac{d x^{k}}{d t}+b_{j k}^{i}(x, t) \frac{d x^{j}}{d t} \frac{d x^{k}}{d t}+c_{j}^{i}(x, t) \frac{d x^{j}}{d t}+d^{i}(x, t)=0
$$

is reducible by a change of variables $\left(t, x^{i}\right) \rightarrow\left(\bar{t}, \bar{x}^{i}\right)$ to the differential system

$$
\frac{d^{2} \bar{x}^{i}}{d \bar{t}^{2}}=0
$$


if, and only if, for $i>3$ the Weyl projective tensor in the space $\left\{x^{i}, t\right\}$ of the associated projective connection with components

$$
\begin{aligned}
\Pi_{j k}^{i} & =b_{j k}^{i}-\frac{1}{n+1}\left(b_{j k}^{m} \delta_{m}^{i}+b_{m k}^{m} \delta_{j}^{m}\right) \\
2 \Pi_{t j}^{i} & =c_{j}^{i}-\frac{1}{n+1} c_{m}^{m} \delta_{j}^{i} \\
\Pi_{j k}^{t} & =-a_{j k} \\
\Pi_{t t}^{i} & =d^{i} \\
\Pi_{t j}^{t} & =-\frac{1}{n+1} b_{m j}^{m} \\
\Pi_{t t}^{t} & =-\frac{1}{n+1} c_{m}^{m}
\end{aligned}
$$

vanishes. The corresponding transformation involves $n(n+2)$ arbitrary constants.

Comparing (88) with (89) we find:

$$
a_{j k}=0, b_{j k}^{i}=0, c_{j}^{i}=0, d^{i}=\varepsilon \omega^{2}(t) x^{i} .
$$

It follows that for these values of the coefficients $a_{j k}, b_{j k}^{i}, c_{j}^{i}, d^{i}$ the only non vanishing component of the projective connection is:

$$
\Pi_{t t}^{i}=\varepsilon \omega^{2}(t) x^{i}
$$

A standard computation shows that the Weyl projective tensor vanishes. Hence the time dependent $n$ dimensional harmonic oscillator is equivalent to the free particle moving in the $n$ dimensional space whose Lie symmetry algebra is 24] the $s l(n+2, R)$. We note that the result holds for any function $\omega^{2}(t)$ hence we arrive at Leach's conclusion that the Lie point symmetry algebra for any linear system in $n$-dimensions is the $\operatorname{sl}(n+2, R)$.

\section{References}

[1] Olver P J 1986 Application of Lie groups to differential equations (Springer Graduate texts in Mathematics, New York: Springer)

[2] Stephani H 1989 Differential Equations: Their Solutions using Symmetry (Cambridge University Press)

[3] Aminova A V and Aminov N A 2006 Sbornic Mathematics 197951

[4] Aminova A V and Aminov N A 2010 Sbornic Mathematics 201631

[5] Feroze T, Mahomed F M and Qadir A 2006 Nonlinear Dynamics 4565

[6] Tsamparlis M and Paliathanasis A 2010 Nonlinear Dynamics 62203

[7] Fredericks E, Mahomed F M, Momoniat E and Qadir A 2008 Computer Physics Comm. 179438

[8] Sen T 1987 Phys. Lett. A 122327

[9] Damianou P A and Sophocleous C 1999 J. Math. Phys. 40210 
[10] Karasu A and Yildirim H 2002 J. Nonlinear Math. Phys. 9475

[11] Leach P G L and Karasu A 2004 J. Nonlinear Math. Phys. 11269

[12] Moyo S and Leach P G L 2002 J. Phys. A 355333

[13] Leach P G L 1981 J. Math. Phys. 22679

[14] Abraham - Shrauner B 1990 J. Math. Phys. 311627

[15] Athorne C 1991 J. Phys. A 241385

[16] Kehagias A and Kofinas G 2004 Class. Quantum Grav. 213871

[17] Bertacca D, Matarrese S and Pietroni M 2007 Mod. Phys. Let. A 22382893

[18] Basilakos S and Lukes - Gerakopoulos G 2008 Phys. Rev. D 781550

[19] Russo J G 2004 Phys. Lett. B 600185

[20] Damianou P A and Sofokleous C 2004 Nonlinear Dynamics 363

[21] Lutzky M 1978 J. Phys. A 11249

[22] Leach P G L 1980 J. Phys. A 131991

[23] Cervero J M and Villarroel J 1984 J. Phys. A 171777

[24] Prince G E and Eliezer C J 1980 J. Phys. A 13815 